\title{
Modelling the air-gap field strength of electric machines to improve performance of haptic mechanisms
}

Conference or Workshop Item

Accepted Version

Harwin, W. S. (2018) Modelling the air-gap field strength of electric machines to improve performance of haptic mechanisms. In: EuroHaptics 2018: Haptics: Science, Technology, and Applications, 13-17 June, Pisa, Italy, pp. 94105. Available at http://centaur.reading.ac.uk/77507/

It is advisable to refer to the publisher's version if you intend to cite from the work. See Guidance on citing.

Published version at: https://doi.org/10.1007/978-3-319-93399-3_9

All outputs in CentAUR are protected by Intellectual Property Rights law, including copyright law. Copyright and IPR is retained by the creators or other copyright holders. Terms and conditions for use of this material are defined in the End User Agreement. 


\section{www.reading.ac.uk/centaur}

\section{CentAUR}

Central Archive at the University of Reading

Reading's research outputs online 


\title{
Modelling the air-gap field strength of electric machines to improve performance of haptic mechanisms
}

\author{
William S. Harwin
}

June $2018^{*}$

\begin{abstract}
The air-gap of electro-magnetic (EM) actuators determines key operating parameters such as their ability to generate force. In haptic devices these parameters are not optimised for the conditions typically seen in operation and include the heat produced in the airgap, the volume of the air-gap, and the intensity and direction of the magnetic field. The relationship between these parameters is complex thus design decisions are difficult to make. This paper considers the role of the radial magnetic field in cylindrical electric motors, a type often used in haptic devices. Two models are derived and compared with experimental measurements. The first model is a closed form solution, the second is a classic Poisson solution to Ampere's equation. These models are shown to be valid for making more general design decisions in relation to haptic actuators, and in particular allow an evaluation of the trade off between the volume of the air-gap, the resulting radial magnetic field and hence heat generated and the resulting forces. electrical machines, electro-magnetic actuators, DC motors, radial magnetic field, diametrically polarised, cylindrical magnet
\end{abstract}

\section{Introduction}

An overlooked aspect of haptic interface research is the demands required of the actuators to deliver the needed forces to the individual. There are few bespoke commercial actuators designed for haptic applications. One exception is the Apple Taptic engine designed to provide haptic feed back in the Apple Watch, iPhone, iPad and MacBook laptops etc. In most other cases haptic interface designers use off the shelf motors and actuators that are often intended for different operating conditions such as gear based transmissions where the actuator should operate at high speeds and low torque in a single direction. In most haptic applications, the preference would be for an actuator that can deliver high force with minimal delay at more modest speeds.

As well as delivering forces with minimal delay, actuators for haptic devices need to transfer kinetic energy into and out of the individual. If we consider an interaction such as simulating the taps of a small (virtual) hammer, we can consider the bounce as nearly an ideal elastic collision. To achieve this in a haptic context requires the movement of the persons hand and arm to be arrested and then accelerated in the opposite direction. This represents a transfer of kinetic energy out of, and back into the person's hand and arm. Managing this energy transferring is

*Proceedings of Eurohaptics 2018, published by Springer 
the responsibility of the haptic actuators, coupled to any motor amplifiers and the power supply. The energy absorbed by the device must either appear primarily as heat loss in the mechanism or actuator, or potentially be transferred back to the power source for storage. Several authors have tried to divert this energy into programmable or auxiliary damping. For example Weir et al. used both passive damping and controlled damping implemented in analogue electronics[12], Harwin et al. used magnetic particle brakes[4] whereas Gosline and Hayward[3] and MohandOusaid et al.[6] used eddy current braking. Although the haptic fidelity and stability are improved, this is at the expense of complexity. It may be possible to reduce this complexity by careful consideration of energy management in a bespoke haptic actuator as it is the actuator that takes the primary burden for delivering the force and managing this energy flow into and out of the person.

Novel actuators may eventually provide useful solutions, and in particular actuators using graphene or low temperature superconductors would have many desirable characteristics for electro-magnetic actuators. High conductivity implies less heat loss through $i^{2} R$ heating, and the thermal properties of graphene would make the management of any of this undesirable heat much easier. However more research will be needed to address difficulties with the fabrication and assembly of graphene components before this material can be applied in the production of efficient commercial actuators for haptic applications. Similarly, although superconducting materials would allow high currents and high magnetic fields to be produced to deliver the necessary forces in a haptic actuator, current materials have a significant overhead in terms of thermal management that would need to be addressed.

Adapting off-the-shelf actuators to haptic devices usually mandates a high efficiency transmission to deliver meaningful forces onto the person[10, 11]. The most common approach is to use a backdrivable transmission principal that assumes that there is a loss-less relation between the motor torques and the endpoint forces[2]. In most 'backdrive' mechanisms the motor spends time operating 'stalled' when the motors do not move, yet need to provide a significant torque. Limitations of off-the-shelf components to operate in these conditions result in haptic devices with an inability to portray hard contact without a 'spongy' feel. Although accuracy and delays in sensing, and the dynamics of the motor and drive electronics all contribute to these problems, this paper focuses on the motor air-gap and the potential for managing energy (including heat) in that space.

A calculation derived in [5] (eq 2) shows the importance of energy management in the air-gap of an electromagnetic actuator intended for haptic interfaces. In an ideal electrical machine with an air-gap volume $V$ it can be shown[5] that the generated force $F$ is given by

$$
F=\frac{1}{\rho^{\frac{1}{2}}} B \sqrt{P_{\text {loss }}} \sqrt{V}
$$

where $B$ is the magnetic field, and $\rho$ is the resistivity of the armature wire and $P_{\text {loss }}$ is the power lost as heat (i.e. $i^{2} R$ heating).

Thus to maximise the available force requires management of these four variables. This paper will not consider $\rho$, a material property of the armature conductor, nor $P_{\text {loss }}$, a necessary consequence of generating high forces, rather it will focus on the inter relationship between air-gap volume $V$ and available magnetic field $B$.

Equation 1 can also be written in terms of the power loss i.e. $P_{\text {loss }}=\frac{F^{2} \rho}{B^{2} V}$. It is then evident that a reduction in this power can be achieved by increasing the magnetic field and the air-gap volume. However these two variables are coupled so for any given magnetic material is there a benefit to increasing the volume of the air-gap given that there will inevitably be a reduction of the magnetic field across the air-gap? But if air-gap volume can be increased there may be 
a secondary benefit in terms of the design of mechanisms to allow efficient extraction of heat from the armature and hence allow for higher actuator forces[5].

The paper will only consider actuators with two pole cylindrical magnets that are diametrically polarised as these are most common in the small DC electric motors used in devices such as the Sensible/3D systems Phantom range, the Novint Falcon, Entact robotics W5D etc.

\section{Theory}

Modelling the magnetic field in a motor air-gap is non-trivial usually requiring a piece-wise solution to the Maxwell equations such as the method of finite element analysis. However simply models are possible and may be of value in gaining a better understanding of the conditions that must be optimised to improve the performance of electro-magnetic actuators for use in haptics. A close form solution to Ampere's non time varying equation $\nabla \times \vec{H}=\vec{J}$ is explored in section 2.1. This equation can be used to get a first approximation of the trade-off between air-gap volume and magnetic field. Section 2.2 overviews a common method used to simplify Ampere's and Gauss's equations for 2 dimensional analysis. The relationship of these results to experimental measurements is given in section 3 and 4 . Discussions and conclusions follow.

\subsection{A two dimensional closed form estimation of the magnetic field in a diametrically polarised cylindrical magnet.}

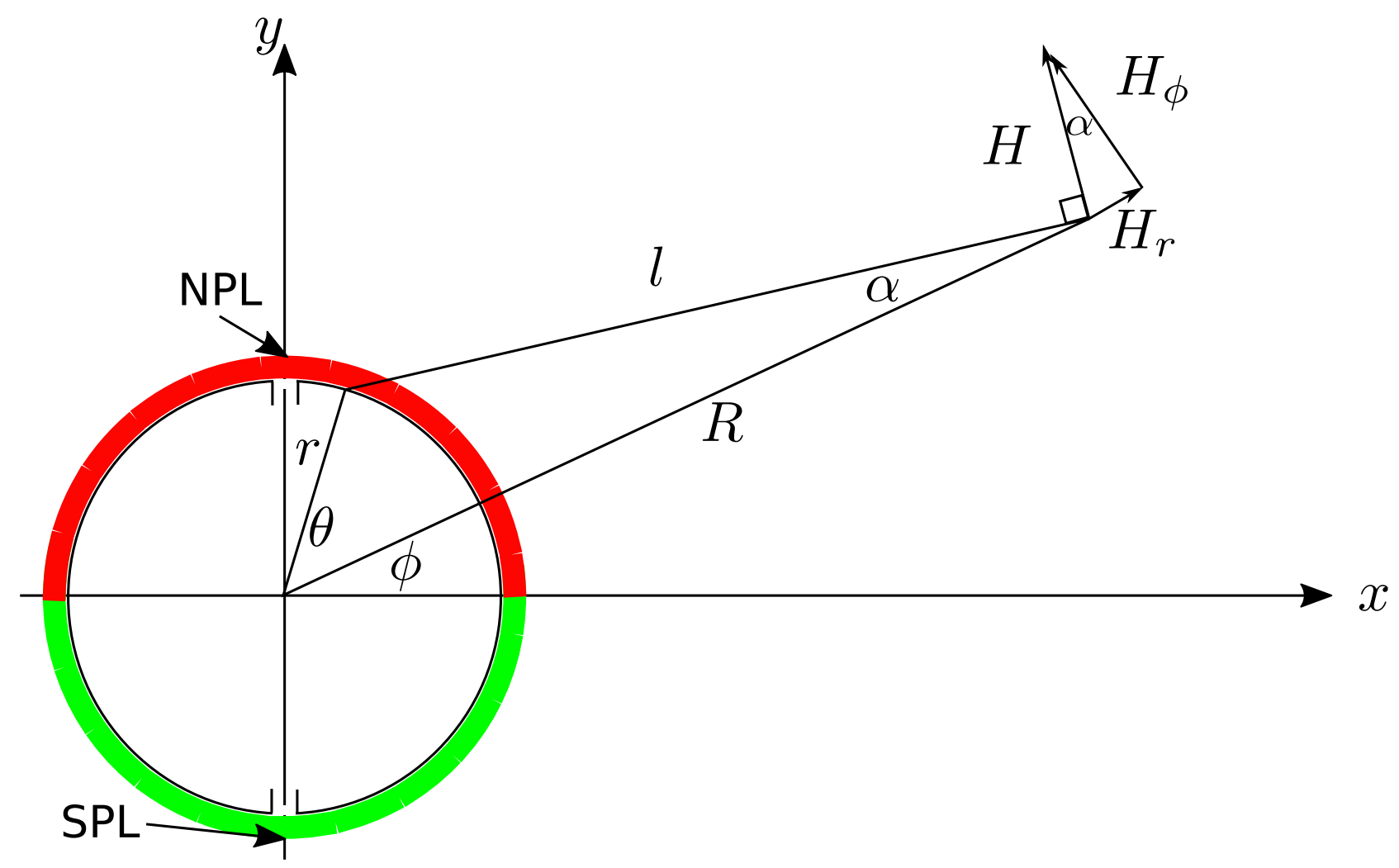

Figure 1: Calculation of the magnetic field of a diametrically magnetised cylindrical magnet. A north pole line (NPL) and a south pole line (SPL) can be considered as the intersection between the magnet and the $y-z$ plane 
A simplified two dimensional version of a static magnetic field for a two pole cylindrical magnet polarised along a radius can be found in closed form. This type of magnet is commonly used in cylindrical motors for haptic applications. As typical of this type of solution we can exploit symmetries in the solution, in particular the field will be symmetric with respect to the $x-z$ plane shown in figure 1. Although the material properties of the cylindrical magnet as characterised by first order reversal curves or second quadrant B-H hysteresis curves could be considered, these do not adapt easily to more general modelling. The approach used in this paper is to characterise the magnetic material properties by a notional surface current representing the underlying causes of the field. In figure 1 this surface current will flow into the paper $(-z)$ for the region $-\pi / 2<\phi<\pi / 2$ and reverse to be out of the paper for the region $\pi / 2<\phi<3 \pi / 2$. This surface current results in the classic concept of a north and a south pole. The location of the peak radial magnetic field occurs along two magnetic poles lines (north pole line - NPL and south pole line - SPL as indicated in figure 1). An instantaneous reversal of current at these two pole lines is initially assumed although this is later relaxed to avoid a singularity in the solution.

With these assumptions we can calculate the radial field at a distance $R$ from the centre of the magnet by considering the current contributions from all the surface currents by integrating $\theta$ through a full circle noting the current reversals as the angle $\phi+\theta$ crosses the $y$-axis.

\subsubsection{Radial field.}

The static magnetic field strength $H$ due to a straight line current $I d \theta$ flowing down the length of the cylinder and situated a distance $l$ from $r d \theta$ can be approximated from Ampere's equation as

$$
H 2 \pi l=I
$$

This field must ensure that the cross product is maintained so that the direction of $H$ must be perpendicular to both the line current in the $z$ direction, and the vector pointing along the measurement $l$. By considering the radial component of $H$ as $H_{r}=H \sin \alpha$ we can then identify the field due to each current element and integrate this equation to compute the field due to the cylindrical magnet.

From figure 1 it is possible to show that the radial magnetic field will be

$$
H_{r}=\int_{-\pi / 2-\phi}^{\pi / 2-\phi} \frac{I}{2 \pi} \frac{\sin \alpha}{l} d \theta+\int_{\pi / 2-\phi}^{3 \pi / 2-\phi} \frac{-I}{2 \pi} \frac{\sin \alpha}{l} d \theta
$$

Using the geometrical relationships $r \sin \theta=l \sin \alpha$ and $l^{2}=(R-r \cos \theta)^{2}+(r \sin \theta)^{2}$, and using the negative sign on the current to reverse the limits of the second term in equation 1 these two integrals can be solved as

$$
H_{r}=\frac{I}{\pi} \int_{-\pi / 2-\phi}^{\pi / 2-\phi} \frac{r \sin (\theta)}{(R-r \cos \theta)^{2}+(r \sin \theta)^{2}} d \theta
$$

On integration this becomes

or

$$
H_{r}=\left.\frac{I}{2 \pi R} \ln \left(R^{2}+r^{2}-2 r R \cos \theta\right)\right|_{-\pi / 2-\phi} ^{\pi / 2-\phi}
$$

$$
B_{r}=\frac{\mu I}{2 \pi R} \ln \left(\frac{R^{2}+r^{2}+2 R r \sin (\phi)}{R^{2}+r^{2}-2 R r \sin (\phi)}\right)
$$

A similar process can be used to compute the tangential field, but it is not necessary to derive this as it does not contribute to the ability of a cylindrical motor to produce torque. 


\subsubsection{Adjustment for modelling the field at the pole lines.}

The calculation of the magnetic field at the two pole lines poses a problem when instantaneous current reversal at the pole lines is assumed. If the radial field at the surface of the magnet is needed along this line the solution is infinite because the sin terms in equation 3 take on the values \pm 1 and the equation reduces to

$$
B_{r}^{\prime}=\frac{\mu I}{2 \pi R}\left(\ln (R+r)^{2}-\ln (R-r)^{2}\right)
$$

Where $B_{r}^{\prime}$ is the radial field along the pole line. Thus there is a singularity when trying to calculate the field along the pole lines at the surface of the cylinder when a division by zero occurs. The obvious solution is to allow a space along the pole line where the surface current is assumed to be zero. This is readily done by changing the integration limits of equation 2 thus

$$
H_{r}=\frac{I}{\pi} \int_{-\pi / 2-\phi+\Delta}^{\pi / 2-\phi-\Delta} \frac{r \sin (\theta)}{R^{2}+r^{2}-2 r R \cos \theta} d \theta+\frac{I}{\pi} \int_{-\pi / 2-\phi-\Delta}^{\pi / 2-\phi+\Delta} \operatorname{ditto} d \theta
$$

hence

$$
B_{r} \frac{2 \pi R}{\mu I}=\ln \frac{R^{2}+r^{2}-2 R r \sin (\phi+\Delta)}{R^{2}+r^{2}+2 R r \sin (\phi-\Delta)}+\ln \frac{R^{2}+r^{2}-2 R r \sin (\phi-\Delta)}{R^{2}+r^{2}+2 R r \sin (\phi+\Delta)}
$$

where $2 \Delta$ now represents the angle at the two pole lines where there is no surface current.

\subsection{Air-gap field estimation using 2D numerical solutions to Maxwell equations}

The theoretical formula described in section 2.1 does not account for the role of the casing in motor design, that helps to intensify the field in the air gap.

An alternative way to estimate this magnetic field is to consider 2D solutions to the Maxwell equations. The following method is well described in standard text books such as [8][9].

The approach used here is to assume that $A_{z}$ (magnetic vector potential) and $J_{z}$ (current density) are scalar and represent the z value of their $3 \mathrm{D}$ equivalents. Assume $\vec{H}, \vec{B}$, the magnetic field strength, and magnetic flux density are $2 \mathrm{D}$ vector fields in the $\mathrm{x}-\mathrm{y}$ plane. It is then possible to reconfigure the static version of Ampere's equation $(\nabla \times \vec{H}=\vec{J})$ as $\nabla \cdot C \vec{H}=J_{z}$ where C is the matrix

$$
C=\left[\begin{array}{cc}
0 & 1 \\
-1 & 0
\end{array}\right]
$$

C serves a similar function to the 3D curl operation $\nabla \times$.

Likewise the gauge equation $\vec{B}=\nabla \times \vec{A}$ that ensures flux conservation $\nabla \cdot \vec{B}=0$ can be rewritten for two dimensional solutions as $\vec{B}=C \nabla A_{z}$. Finally the relationship $\vec{B}=\mu \vec{H}$ where $\mu$ is a property of the material will be used throughout, including an assumption that it will apply to the hard magnetic material of the motor core magnet. Instead the magnetising strength of this material will be modelled as a surface current $J_{z}$ that reverses at the half way line between the two pole lines (NPL/SPL).

These assumptions allow the solution to be written as a Poisson problem

$$
-\nabla \cdot\left(\frac{1}{\mu} \nabla A_{z}\right)=J_{z}
$$

By adding boundary conditions, this class of problem can be solved with tools such as the Matlab PDE (partial differential equation) toolbox. 


\subsubsection{Numerical simulation of the air-gap of a cylindrical motor.}

Figure 2 shows a solution as a quarter representation of the field in the air-gap of a cylindrical motor. Dimensions are approximately those of the Maxon RE25 motor, that is an air-gap width of $2 \mathrm{~mm}$ and a magnet radius of $8.5 \mathrm{~mm}$. This figure shows the field as a scaled direction vector. The estimated radial field strength results of this simulation are plotted along with experimental measurements in figure 5 .

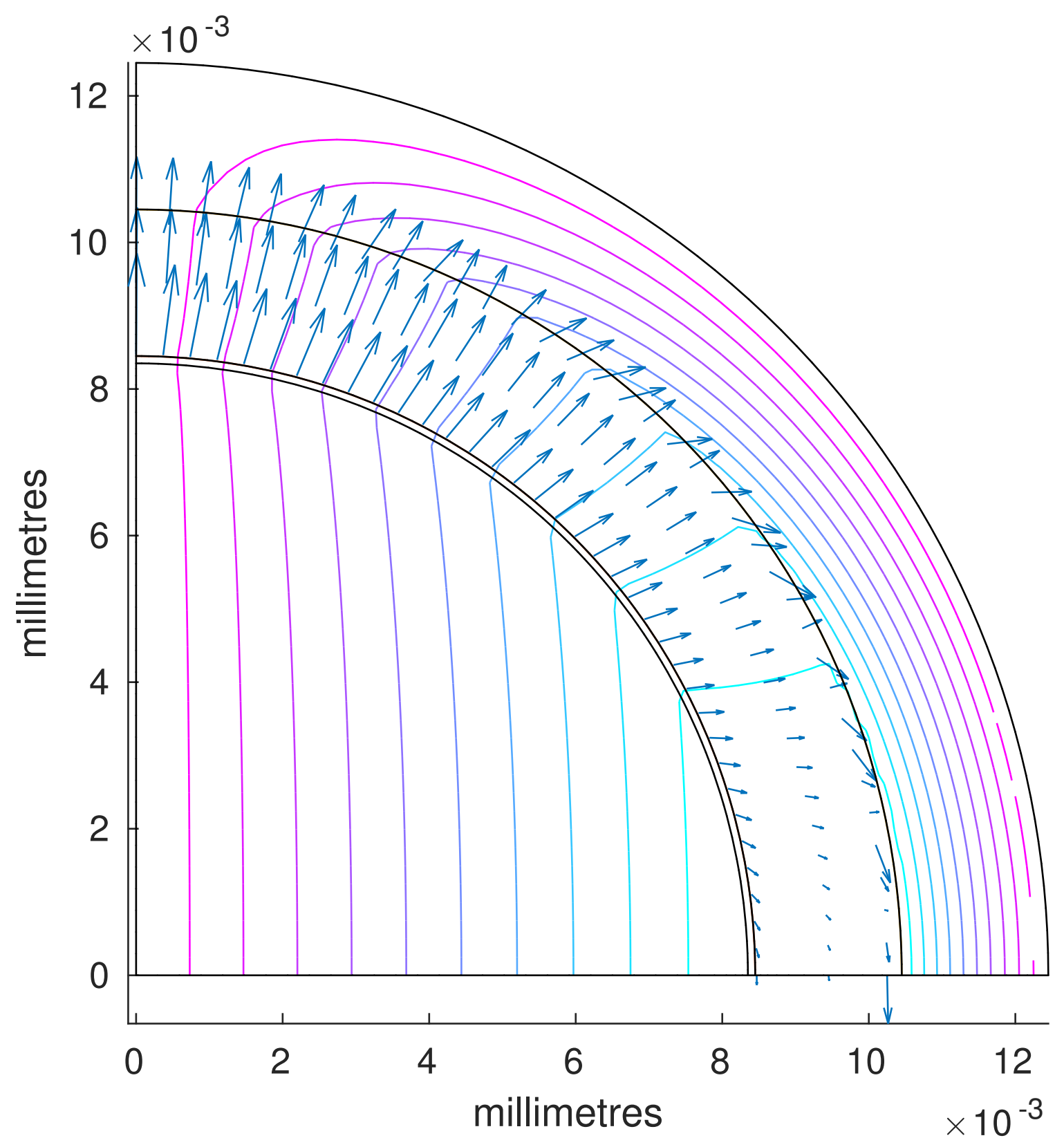

Figure 2: 2D simulation of the field in the air-gap of a Cylindrical magnet motor such as the Maxon RE25 using Matlab PDE toolbox. Arrows show the direction and magnitude of the magnetic field in the air-gap 


\section{Experimental measurement of the radial field of a cylin- drical magnet}

The radial field was measured on two cylindrical magnet configurations to assess the validity of the proposed models. The first configuration was a cylindrical magnet with a diameter of $23 \mathrm{~mm}$ that was sufficiently removed from magnetic material to assume a free space condition. The second was the air-gap of a partially disassembled Maxon RE25 motor. The field was measured using a GMET H001 Gauss meter (CERMAG Ltd) with a 1-3000 Gauss range and a 1 Gauss resolution using a transverse (thin) probe.

All experiments were done using an indexing head at 9 degree increments - this was for convenience since this particular indexing head had a 40:1 gear ratio.

\section{Results}

\subsection{Field of a cylindrical magnet}

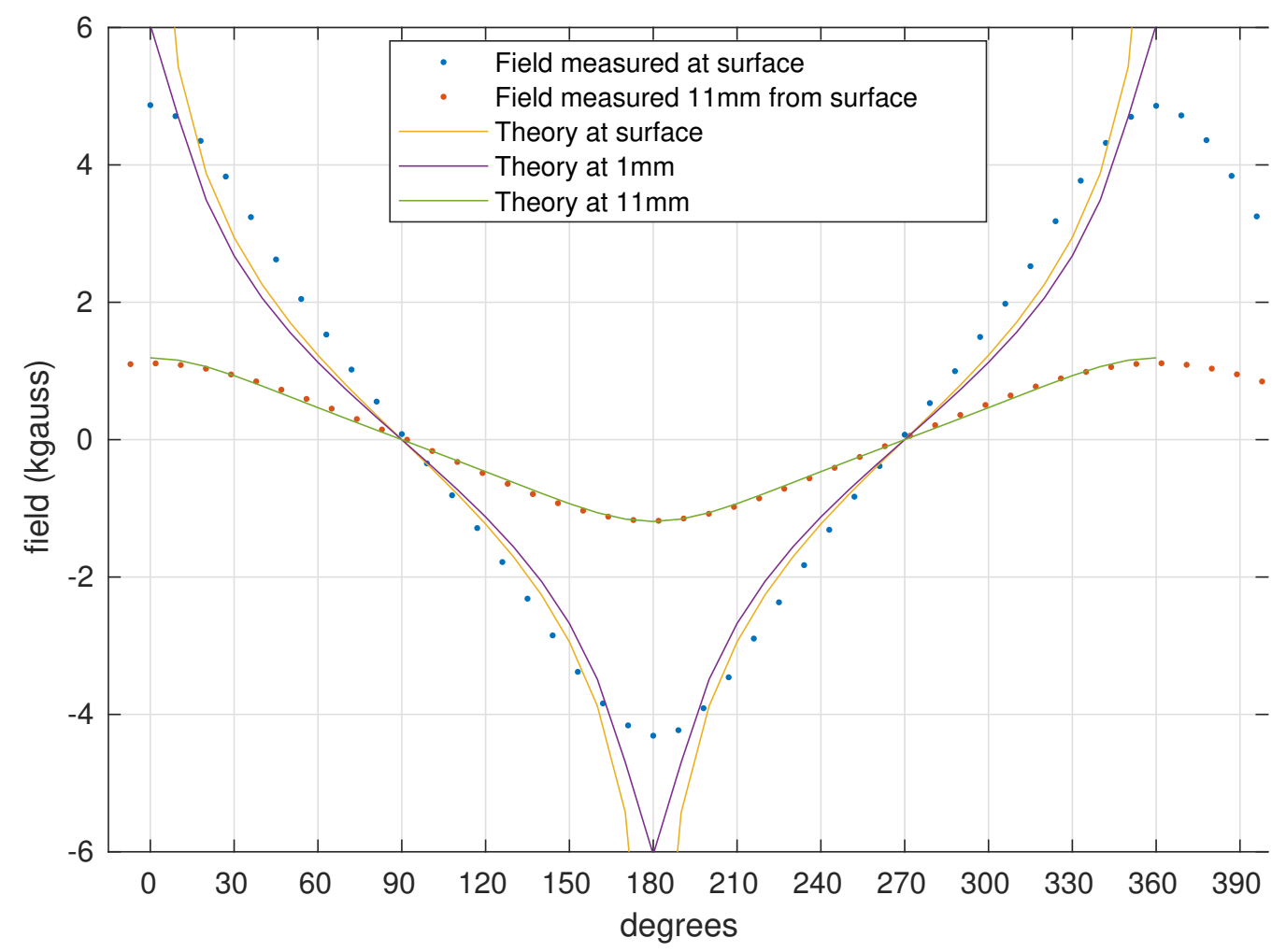

Figure 3: Theoretical radial field vs experimental results for a free standing 23mm diameter cylindrical magnet. The current density is assumed to be $6.6 \times 10^{4} \mathrm{~A} \mathrm{rad}^{-1}$

Figure 3 shows the measured radial field of the isolated cylindrical magnet. Also shown in the graph are the theoretical result as predicted by equation 3. The figure clearly shows that the model works well away from the surface of the magnet and away from the pole lines. The model does not predict the field well along the two pole lines. This is as expected by consideration of equation 3 .

Figure 4 shows that the revised model as described by equation 4 is a better fit to the experimental data. This supports the premise that the surface current density may not change 


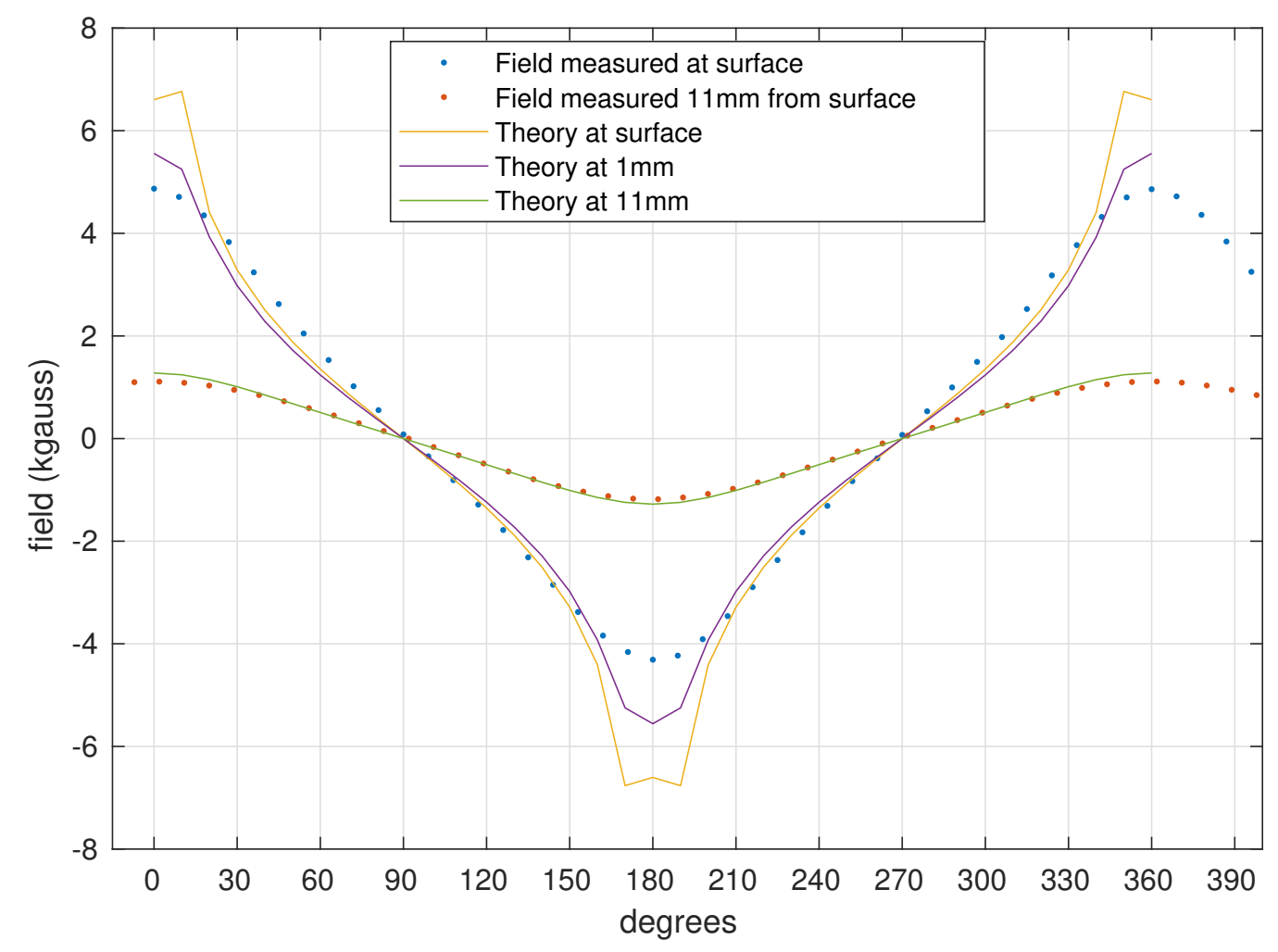

Figure 4: Theoretical radial field vs experimental results for a free standing $23 \mathrm{~mm}$ diameter cylindrical magnet. Theory assumes a region of about 15 degrees at each pole where magnetising current density is assumed to be zero. Elsewhere the current density is assumed to be $3.6 \times 10^{4} \mathrm{~A} \mathrm{rad}^{-1}$

instantaneously along the pole line. This may be a consequence of the magnetisation procedure or a material limitation. Like the simpler model, the prediction of field away from magnet surface and away from the pole line is good.
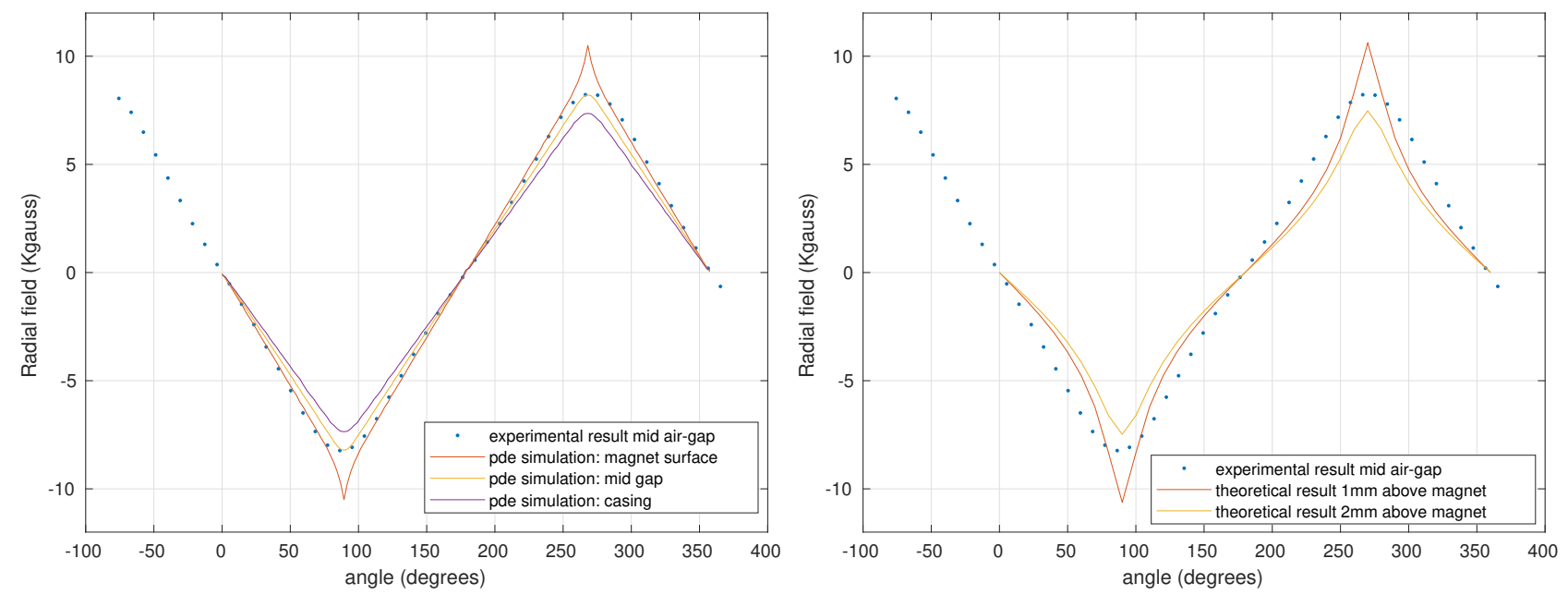

Figure 5: Measured and computed radial magnetic field in the air-gap of a Maxon RE25 motor (magnet diameter $8.5 \mathrm{~mm}$ air-gap $2 \mathrm{~mm}$ ). Experimental results are common to both graphs as described in section 3. Left graph shows the prediction from a numerical solution to the simplified Ampere equations. Right graph shows the prediction made using equation 3 of a free standing cylindrical magnet 
Table 1: Numerical estimates (via Matlab PDE toolbox) of the mean and maximum magnetic field in the mid air-gap of a theoretical motor with a 2-pole cylindrical magnet with a $20 \mathrm{~mm}$ diameter. The $2 \mathrm{~mm}$ air-gap results are directly comparable with the results shown in figure 5 (left)

\begin{tabular}{rrr}
\hline $\begin{array}{r}\text { air-gap } \\
\mathrm{mm}\end{array}$ & $\begin{array}{r}\text { max field } \\
\text { kGauss }\end{array}$ & $\begin{array}{r}\text { mean field } \\
\text { kGauss }\end{array}$ \\
\hline 2 & 8.213 & 4.236 \\
4 & 4.099 & 2.171 \\
6 & 2.648 & 1.435 \\
8 & 1.910 & 1.056 \\
\hline
\end{tabular}

Figure 5 shows the radial field as measured at the mid radius in the air-gap of a Maxon RE25 motor. The comparison between the measured results and the numerical solution to the simplified Ampere equation can be seen in the left hand graph, and the right hand graph overlays a computed value of the radial magnetic field as given in equation 3 . Table 1 uses the results of the 2D simulation described in section 2 solved with the Matlab PDE toolbox to estimate the mid air-gap field of a cylindrical magnet with enclosure and shows the trade-off between air-gap volume and field strength.

\section{Discussion}

Both the partial differential equation based numerical model and the closed form model of a cylindrical magnet field are viable for modelling the field in the air-gap of a cylindrical motor and hence evaluating the potential for better motor efficiency for a haptic application. It is evident that the numerical solution based on a 2D solution to Ampere's equation provides better accuracy, particularly at the pole lines, and can include the effects of the casing in enhancing the field, however the closed form with adjustment for the pole line singularity may be acceptable and is much simpler to calculate.

Using these methods the loss in field strength as the diametrical air-gap radius is increased is shown to be roughly in proportion (Table 1), so increasing the air-gap volume is countered (as expected) by the loss in magnetic field strength. This suggests that management of heat in an actuator designed specifically for haptic applications will need to consider more than just an increase in the air-gap volume to improve efficiency, and more consideration will be needed to ensure that heat is conducted away from the air-gap. However it would seem feasible to increase the air-gap volume and accept a lower magnetic field strength given the potential that may allow for better heat management.

Future work will now need to consider thermal modelling (e.g. [1, 7]) in addition to field modelling to optimise electrical machines for haptic applications. As has been shown, the modelling of this field just over the pole lines is difficult so the calculations could use an offset, possibly by 15 to 30 degrees where the surface current is assumed to be zero so as to get best use out of the magnetic field models. 


\section{Conclusion}

The two models of the radial magnetic field of a cylindrical magnet presented in this paper have been validated against experimental results for both an exposed cylindrical magnet, and the air-gap of a typical motor used in haptic applications. In both cases there is good agreement between the models and the measured radial magnetic field. The derivation of a robust and believable framework for the field in the air-gap of a cylindrical motor will allow better evaluation of the inter-relationships between the volume of an actuator air-gap and the resulting field and hence ability to produce continuous and controllable forces via the motor. In turn this may enable better design decisions about the managing the heat generated by the armature and hence the ability of the actuator to produce a sustained force. The understanding the relationships between the ability of the actuator to generate high sustained forces and the air-gap volume will require careful evaluation for haptics applications as the $i^{2} R$ heat generated by the armature is a necessary condition for force generation in an electro-magnetic haptic actuator. Increasing the air-gap volume may provide a rational approach to improving the extraction of this heat without compromising the actuator performance.

\section{Acknowledgements}

With thanks to Peter Tolson and Mike Charij for assisting with the experiments and to Maxon motors for providing old stock motors.

\section{References}

[1] Paul Buckley et al. "Effects of thermal protection methods on haptic perception". In: World Haptics Conference (WHC), 2011 IEEE. IEEE. 2011, pp. 143-148. DOI: 10.1109/ WHC.2011.5945476.

[2] C.R. Carignan and K.R. Cleary. "Closed-Loop Force Control for Haptic Simulation of Virtual Environments". In: haptics-e.org 1.2 (2000). URL: http://www.haptics-e.org/ Vol_01/index.html.

[3] Andrew HC Gosline and Vincent Hayward. "Dual-channel haptic synthesis of viscoelastic tissue properties using programmable eddy current brakes". In: The International Journal of Robotics Research 28.10 (2009), pp. 1387-1399.

[4] W.S. Harwin et al. "Design and clinical potential of programmable mechanical impedance". In: Robotica 5 (1998), pp. 523-530. ISSN: 0263-5747. DOI: 10.1017/S026357479800068X.

[5] Rebecca Jarman, Balazs Janko, and William S. Harwin. "Current over-stressing small DC motors to evaluate performance limits of electromechanical actuators for haptic applications". In: 2015 IEEE World Haptics Conference. 2015, pp. 171 -176. DOI: 10.1109/ WHC.2015.7177709.

[6] Abdenbi Mohand-Ousaid et al. "Haptic interface transparency achieved through viscous coupling". In: The International Journal of Robotics Research 31.3 (2012), pp. 319-329.

[7] M. Rios et al. "Thermal performance modeling of foil wound concentrated coils in electric machines". In: 2016 IEEE Energy Conversion Congress and Exposition (ECCE). 2016, pp. 1-8. DOI: 10.1109/ECCE.2016.7855512. 
[8] Sheppard Joel Salon. Finite Element Analysis of Electrical Machines. Published by Springer, 1995. ISBN: 978-0-7923-9594-2. URL: http://books.google.co.uk/.

[9] Gilbert Strang. Computational science and engineering. Wellesley-Cambridge Press, 2007.

[10] William T. Townsend. "The effect of transmission design on force-controlled manipulator performance". Cable efficiency discussed in chapter x 67-77. PhD thesis. MSME: MIT, 1988. URL: https://dspace.mit.edu/bitstream/handle/1721.1/6835/AITR-1054.pdf.

[11] W.T. Townsend and J.K. Salisbury. "The efficiency limit of belt and cable drives". In: Journal of mechanisms, transmissions and automation in design 110.3 (1988), pp. 303307.

[12] D.W. Weir, J.E. Colgate, and M.A. Peshkin. "Measuring and increasing Z-width with active electrical damping". In: Haptic interfaces for virtual environment and teleoperator systems, 2008. haptics 2008. symposium on. IEEE. 2008, pp. 169-175. 